
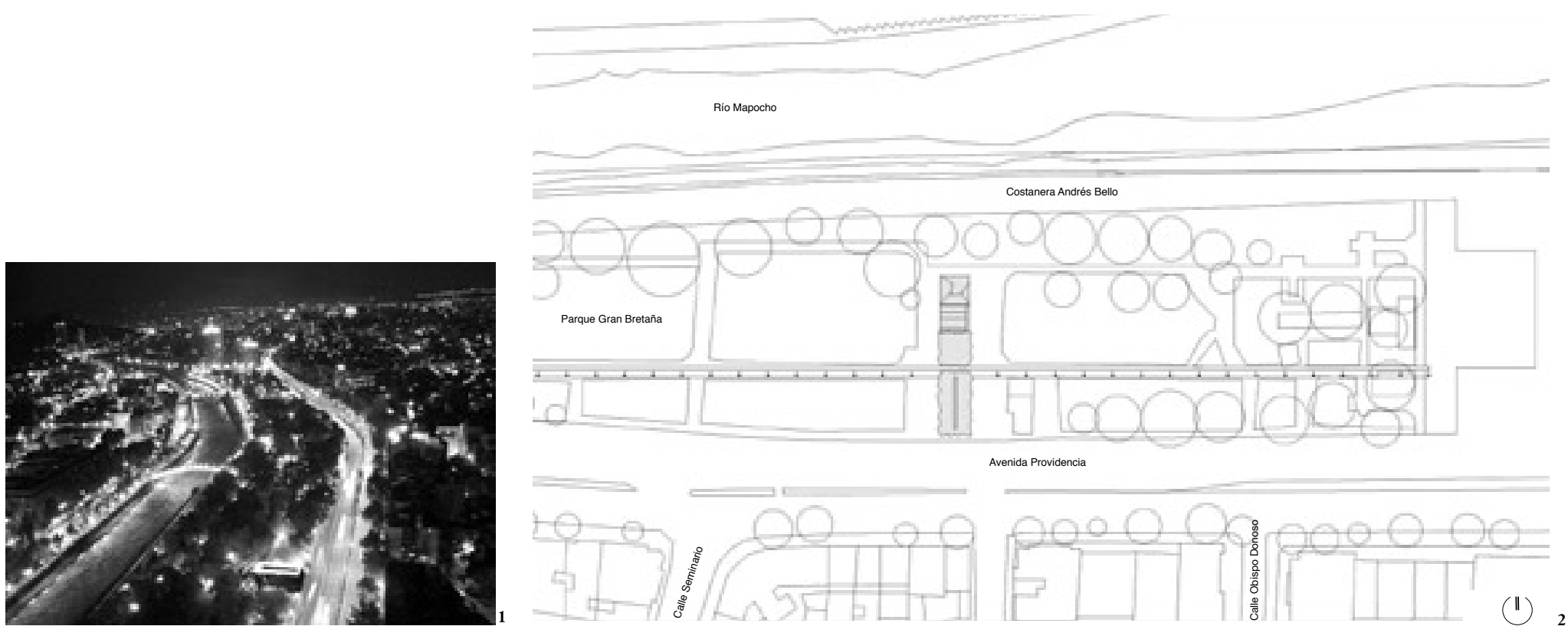

Alejandro Aravena + Charles Murray

\title{
Museo del Vino
}

Bienvenidos al sistema de peregrinaje cultural -o en el peor de los casos, al impensado menàge a trois entre ocio, capitalismo y cultura-. De la misma manera en que las vanguardias de las artes visuales se confunden con formas de expresión despreciadas hasta bace 50 años (Michel Gondry mediante) los temas de la cotidianeidad se cuelan hacia las plazas reservadas a la "alta cultura". ¿Maniobra de los grandes grupos del capital o síntoma de la complejización de una realidad valóricamente homogénea?

Palabras clave: Arquitectura-Chile, museos, vino, salas de exposición, tajamares.

Welcome to cultural pilgrimages -or, in the worst case, an unforeseen ménage a trois of leisure, capitalism and culture. fust as the visual arts vanguards blend into forms of expression despised until 50 years ago (Michel Gondry, thank you), so day-to-day topics slip into the spaces reserved for "bigh culture". Is it an operation by big capitalist groups? Or a sign of the growing complexity of a homogeneous values world? Key words: Architecture-Chile, museums, wine, exhibition halls, embankments.
Este museo es un encargo de la Corporación Cultural Municipalidad de Providencia, con la concurrencia de distintas viñas privadas, y busca remodelar el antiguo Museo de Los Tajamares en el parque Gran Bretaña.

El museo en su estado actual, si bien se trata de un espacio de cierta calidad asociado al descubrimiento de restos de antiguos muros durante las obras de construcción del Metro, es un lugar marginal como galería de exposiciones y con graves problemas de anegamiento.

La Corporación Cultural quiso sacar partido de su ubicación central y recuperarlo como un lugar estratégico en los itinerarios culturales y turísticos de Santiago. Su condición de cava natural dio origen a la idea de transformarlo en un Museo del Vino, programa hasta ahora inexistente en el país.

Ahora bien, ¿Cuáles son los problemas que se deben abordar en este proyecto?

Creemos que son básicamente cuatro:

1. Recuperar la condición natural de los tajamares, reconociendo su valor arqueológico, llevándolos por tanto a su estado original. Se debe además cubrir el foso actual, para evitar las inundaciones que lo afectan en este momento y para darle al mismo tiempo una presencia en el parque. Este espacio cubierto es el que se transformaría en la sala principal del museo, ausente en el museo actual.

2. Atender la relación entre lo que se expone, en este caso el vino, y el sentido por medio del cual se percibe aquello expuesto. Si comparamos esto con una pintura o una escultura, tenemos que la manera última de conocer una obra plástica es por medio del sentido de la vista. En el caso del vino, la manera última de conocer lo expuesto es por medio del sentido del gusto. Entonces, ¿cómo evitar que el museo se transforme en un bar? Proponemos mostrar tantos aspectos como sean posibles del elemento expuesto, desde el cultivo que le da origen hasta otras dimensiones a las que un experto presta atención: color, textura, aroma. Es en este sentido que nos interesa que el Museo del Vino sea un edificio de vino. La idea es que el volumen del acceso esté compuesto por termo- paneles de vidrio al vacío con vino en su interior, que compongan un gran "vitreaux de vino" donde se expongan todas las tonalidades de éste. Cada uno de estos paneles sería una especie de botella plana de $90 \times 270 \mathrm{~cm}$ aportada por las viñas.

3. Todo proyecto inserto en un espacio cualquiera 
1 El museo en el parque Gran la derecha, el rí Mapocio

2 Plano de emplazamiento

3 Sistema de pliegues, superficie

del volumen sur
4 Vista general desde el río

5 Vista del patio norte y

volumen vidriado de acceso

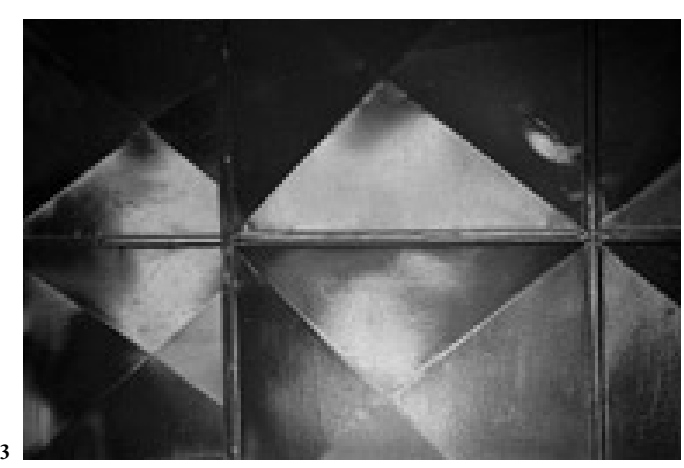

tiene que preguntarse cómo debiera ser su arquitectura; nos ha parecido que lo mejor es dejar que la arquitectura pase a un segundo plano y para ello se han tomado dos caminos: por una parte, las fachadas del museo estarán cubiertas por distintas variedades de vid, siendo la propia materia prima del vino uno de los protagonistas de la exhibición. De hecho, la noción tradicional de elevación norte poniente o sur será reemplazada por la de fachada Cabernet, Merlot o Carmenère por nombrar algunas posibilidades.

Por otra parte, frente al espacio del parque, respondemos con un elemento natural: una piedra que al carecer de medida (la noción de cuántos pisos tiene, por ejemplo, carece de sentido) puede tener presencia sin sacrificar el parque.

4. Más que un problema a resolver, habría que hablar del horizonte del proyecto: de lo que se trata aquí es, por medio de la arquitectura, aparecer en el circuito internacional. Tenemos el ejemplo del Guggenheim de Bilbao o de la participación de la obra de Siza en los destinos turísticos de Portugal. Interesa aclarar que aspiramos a que la intensidad de la obra tenga impacto a escala mundial. De ahí su radicalidad y la táctica que busca apostar por aquello en lo que tenemos ventajas: lo primitivo y lo natural, la piedra y la vid. ARQ
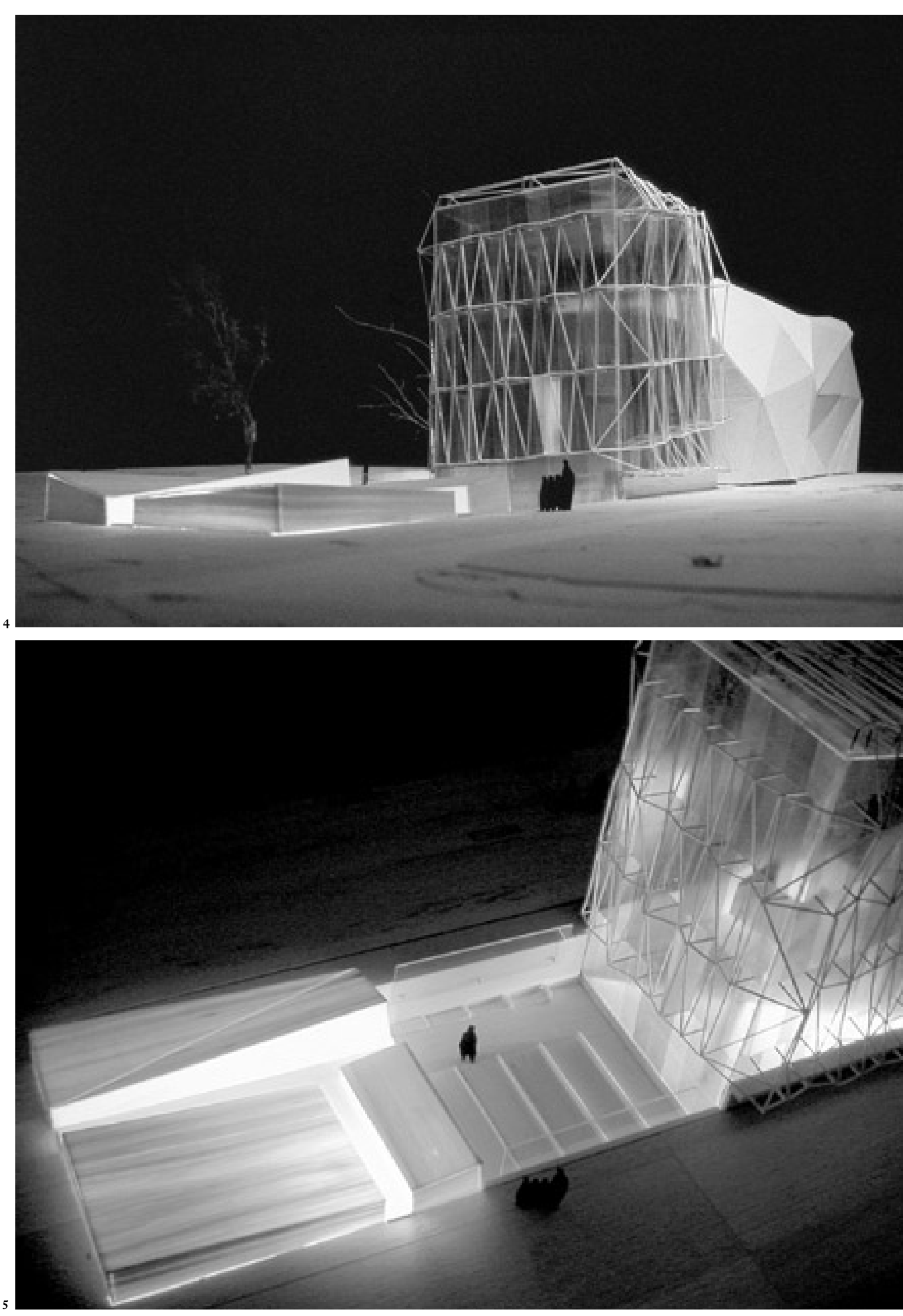


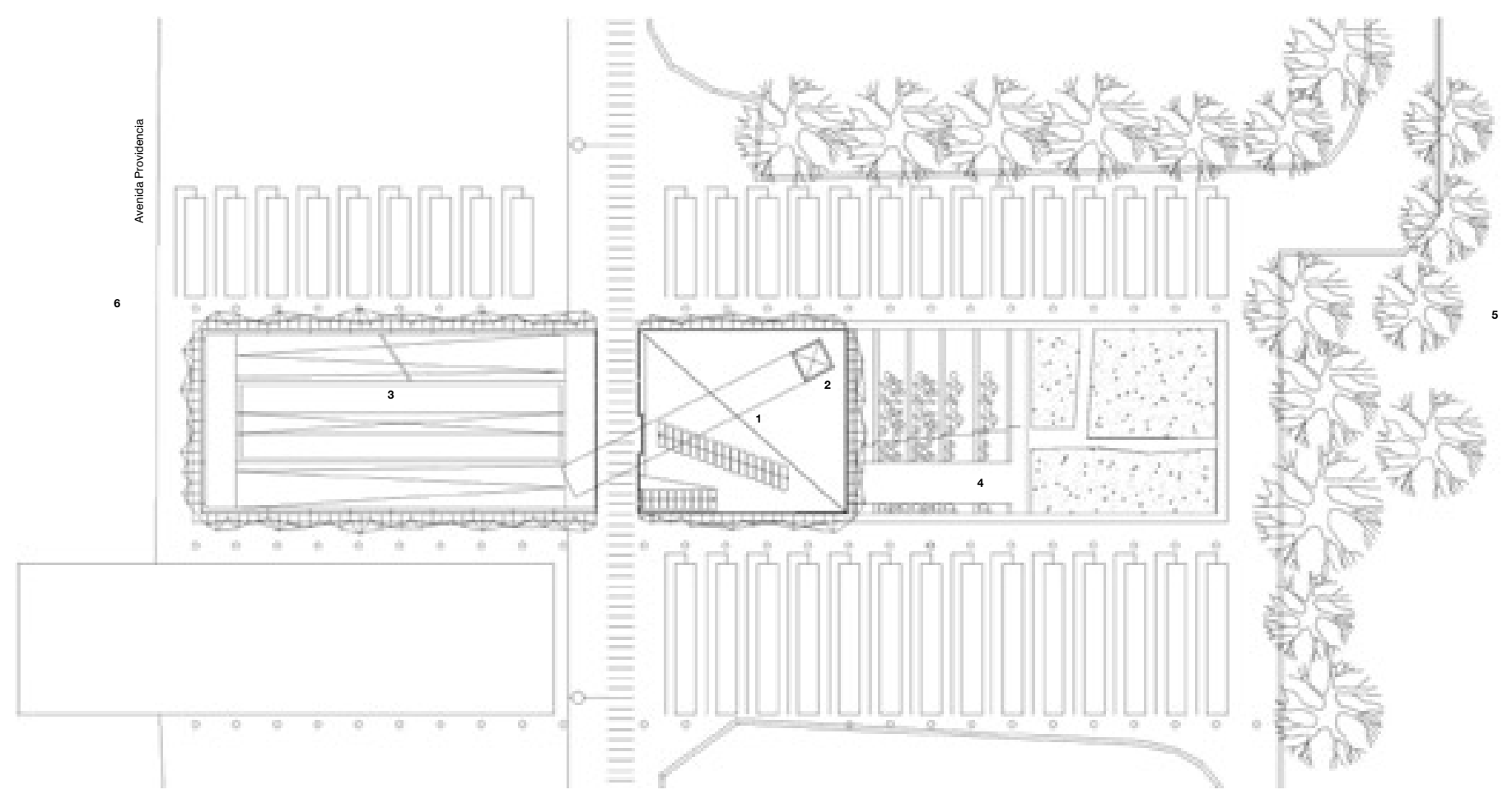

Planta general nivel parque

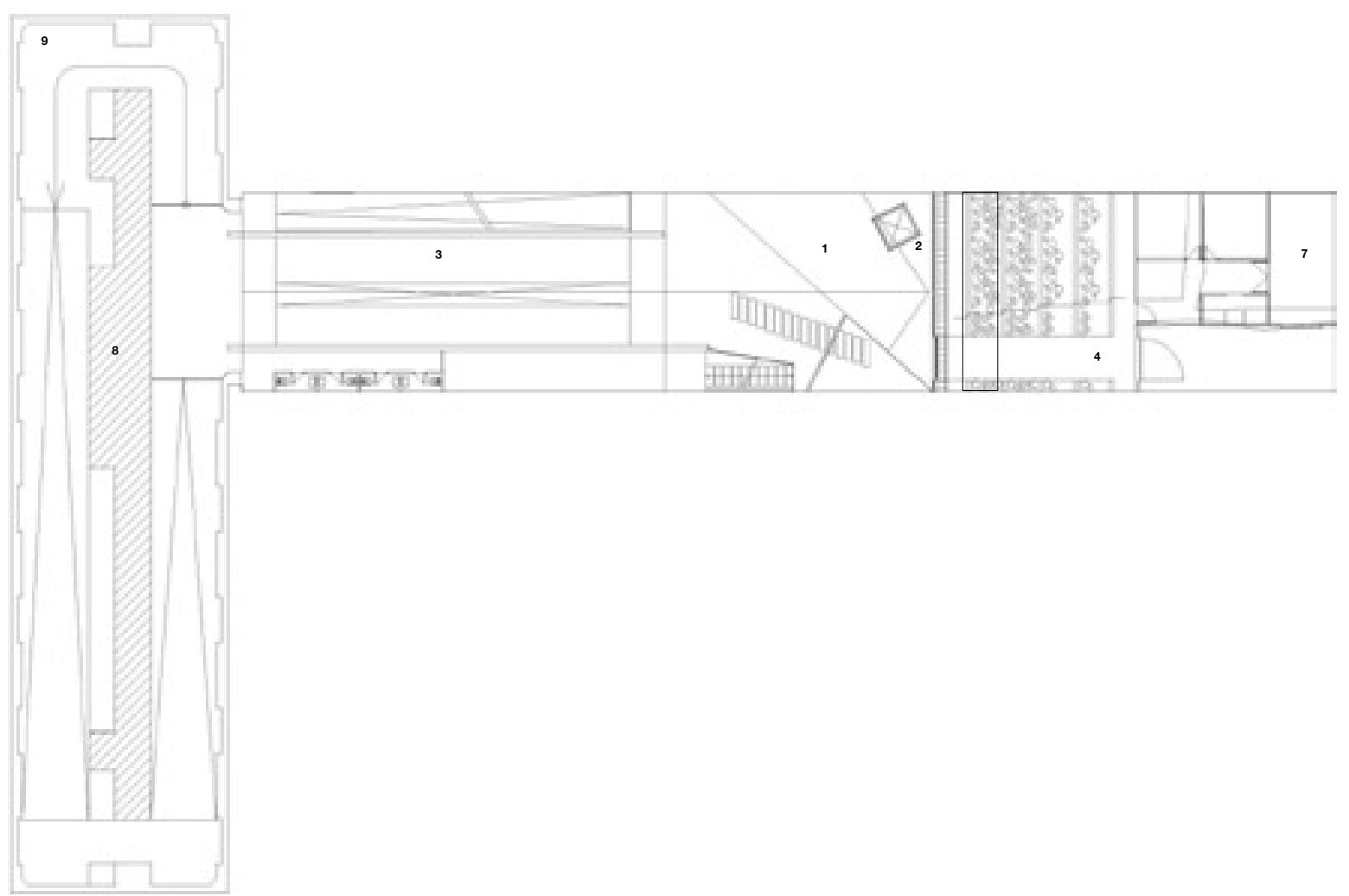

Pabellón norte - hall
Ascensor

Ascensor
Pabellón sur - exposiciones

Jardin Gran Bretana

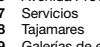

Planta nivel tajamares, subsuelo

ARQ Obras y proyectos Works and projects 
6 y 7 Espacio interior para

exposiciones, volumen sur

8 Vista oriente. A la izquierda,

Avda. Providencia
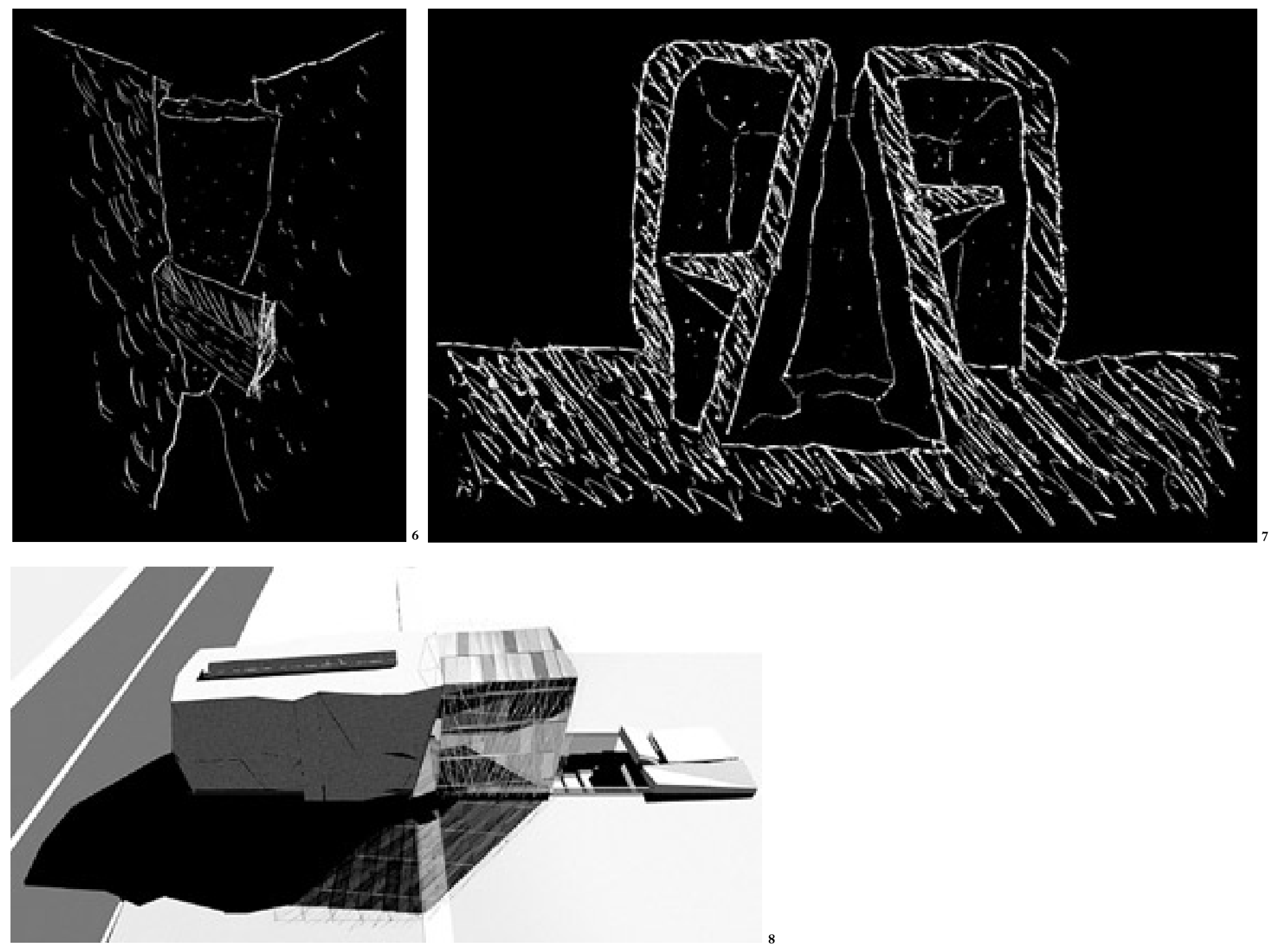

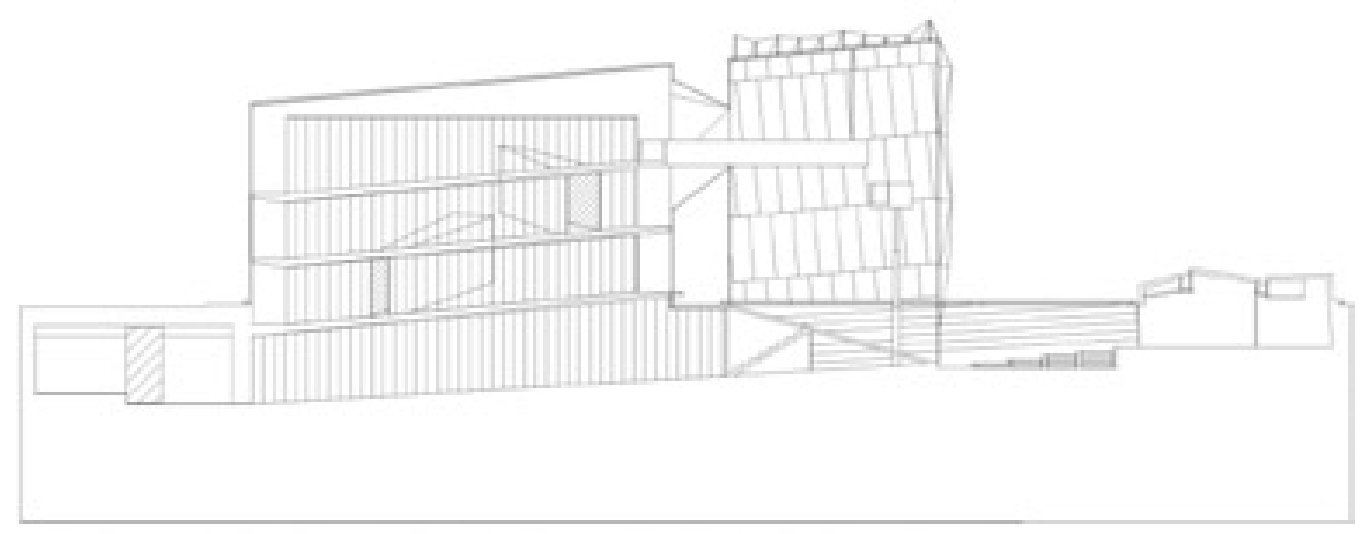

Corte longitudinal

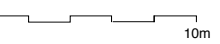

Museo del Vino

Arquitectos Alejandro Aravena + Charles Murray Colaboradores Tarek Ghandour + Juan Isnacio Cerda Ubicación Parque Gran Bretaña, Avda Providencia esq. Seminario, Santiago, Chile

Mandante Corporación Cultural de la I. Municipalidad de Providencia

Materialidad Estructuras de contención de hormisón armado, estructuras de cubiertas de acero, cerramientos de vidrio y vid

Superficie construida $2.000 \mathrm{~m}^{2}$

Año proyecto 2001-2002

Fotografías Alejandro Aravena

Imásenes digitales Danilo Lagos 